\title{
Fracture of Brittle Metallic Glasses: Brittleness or Plasticity
}

\author{
X. K. Xi, ${ }^{1}$ D. Q. Zhao, ${ }^{1}$ M. X. Pan, ${ }^{1}$ W. H. Wang,,${ }^{1, *}$ Y. Wu, ${ }^{2}$ and J. J. Lewandowski ${ }^{3}$ \\ ${ }^{1}$ Institute of Physics, Chinese Academy of Sciences, Beijing 100080, China \\ ${ }^{2}$ Department of Physics and Astronomy, University of North Carolina, Chapel Hill, North Carolina 27599-3255, USA \\ ${ }^{3}$ Department of Materials Science and Engineering, Case Western Reserve University,
}

10900 Euclid Avenue, 44106 Cleveland, Ohio, USA

(Received 25 November 2004; published 1 April 2005)

\begin{abstract}
We report a brittle Mg-based bulk metallic glass which approaches the ideal brittle behavior. However, a dimple structure is observed at the fracture surface by high resolution scanning electron microscopy, indicating some type of "ductile" fracture mechanism in this very brittle glass. We also show, from the available data, a clear correlation between the fracture toughness and plastic process zone size for various glasses. The results indicate that the fracture in brittle metallic glassy materials might also proceed through the local softening mechanism but at different length scales.
\end{abstract}

DOI: 10.1103/PhysRevLett.94.125510

Despite intense investigation, controversy remains about the fracture mechanism of brittle glasses [1-5]. Oxide glasses are the most common example of brittle materials, while the ductile fracture mode is widely observed for a large variety of metallic materials. Kelly et al. [3] concluded that metallic solids deform plastically at crack tips whereas nonmetallic glasses (e.g., silicate glasses) generally support perfectly brittle cracks; the fundamental mechanism of brittle fracture is one of sequential bond rupture. This view was further strengthened by the theory of Rice and Thomson [4] and by the experimental observations of Guin and Wiederhorn [5]. Recently, a new family of Mg-based bulk metallic glasses (BMGs), which approaches the brittleness of silicate glasses when subject to uniaxial tension or three-point bending, has been developed [6]. Unlike the insulating covalent or ionic glasses whose fracture surfaces are quite difficult to investigate by means of high resolution scanning electron microscopy (HRSEM), it is convenient to investigate features of fracture surfaces of conducting BMGs on a nm scale using HRSEM. Therefore, the brittle Mg-based BMGs with notch fracture toughness $K_{C}$ of $2.0 \pm 0.2 \mathrm{MPa} \sqrt{\mathrm{m}}$ (see the details in Refs. [7,8]), which approaches the ideal brittle behavior associated with silicate glasses with $K_{C} \sim$ 0.68 to $0.91 \mathrm{MPa} \sqrt{\mathrm{m}}$ in dry nitrogen gas at room temperature [9], provide a model material for investigating the fracture of brittle glassy metals. It offers an opportunity for a fundamental understanding of fracture behavior of metallic glasses.

The fractures of the BMGs occur near the theoretical strength (about $E / 50, E$ is Young's modulus) [10]. In such systems the initiation and propagation of locally intense shear upon fracture produce the characteristic "vein" pattern in scanning electron microscopy (SEM) images of fracture surfaces [8,11-14]. This extreme local ductility combined with high strength provides one source of the reported high fracture toughness values in these BMGs (e.g., Zr-based BMGs). In notched samples many shear bands initiate and propagate from the notch, leading to
PACS numbers: 62.20.Mk, 61.43.Fs, 81.40.Np

$K_{C}>60 \mathrm{MPa} \sqrt{\mathrm{m}}[8,14]$. It is known that the stress intensity factor $K_{C}$ provides a universal description of the fracture process. If any two systems have the same value of $K_{C}$, the corresponding crack tips will behave in the same way [1]. The availability of various BMGs, with marked differences in toughness and a wide range of $K_{C}[7,10]$, make it possible to correlate the feature of the glassy fracture surfaces with their mechanical properties. The link established between mechanical behaviors and fracture features can assist in elucidating the fracture mechanisms, and in guiding glassy alloy design to alleviate brittleness.

In this Letter, we report the HRSEM observation of the fracture surfaces of the brittle $\mathrm{Mg}_{65} \mathrm{Cu}_{25} \mathrm{~Tb}_{10}$ BMG. The experimental evidence for a $100-\mathrm{nm}$ scale plastic process zone in the fracture surface of the very brittle glass is presented. The local necking topographic structure supports a shear-softening mechanism in the fracture. Analysis of the length scale of the plastic process zone ranging from a $100-\mathrm{nm}$ scale to a $100-\mu \mathrm{m}$ scale in various metallic glasses establishes a clear correlation between the length scale of the plastic process zone and fracture toughness and strength.

The fracture surfaces of cylindrical $\mathrm{Mg}_{65} \mathrm{Cu}_{25} \mathrm{~Tb}_{10} \mathrm{BMG}$ ( $5 \mathrm{~mm}$ in diameter) and other BMGs were prepared by three-point bending fracture of single-edge notched samples on a servohydraulic testing machine under a constant displacement rate at ambient conditions (see the details in Ref. [8]). The size of the specimen necessary for plane strain conditions at the crack tip satisfies the criterion described in Ref. [15]. The rate of strain was chosen so as to have at most one $0.1-\mu \mathrm{m}$ step during the course of fracture where elastic waves induced by sample loading could be eliminated. HRSEM with a resolution of $1.5 \mathrm{~nm}$ was performed on a Philips XL 30 Environmental SEM operated at 5-10 keV. The atomic force microscopy (AFM) was carried out in a Digital Instruments NanoScope IIIa D-3000 AFM.

Figure 1(a) shows the SEM micrograph of the fracture surface of the $\mathrm{Mg}_{65} \mathrm{Cu}_{25} \mathrm{~Tb}_{10}$ BMG. The surface clearly 


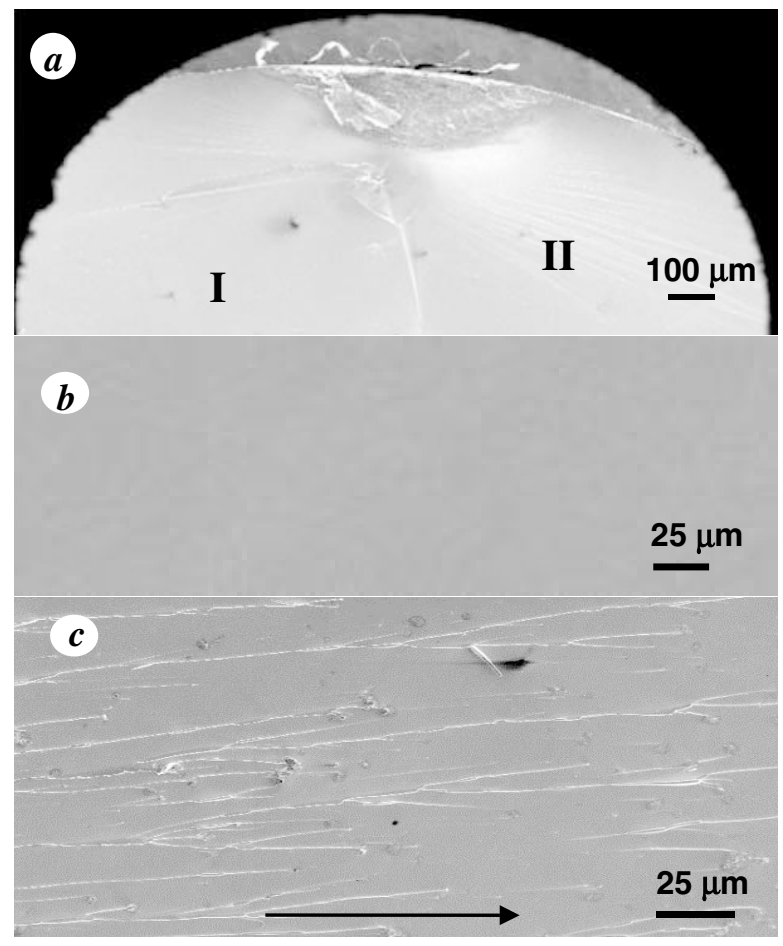

FIG. 1. (a) SEM fracture surface appearance of an amorphous $\mathrm{Mg}_{65} \mathrm{Cu}_{25} \mathrm{~Tb}_{10}$ alloy cylinder showing distinct regions, (b) flat mirror zone (I), and (c) mist zone consisting of massive river vein patterned shear bands (II). The arrow shows the crack propagation direction.

shows a typical flat mirror zone (zone I) followed by a mist and shear band branching zone (zone II). The dominant feature under three-point bending is featureless zone I. No obvious crystallization and phase separation were observed in the fracture surface by x-ray diffraction and high resolution transmission electron microscopy. The extent of the mirror region decreased and the severity of hackle increased with increasing the fracture strain rate. This is consistent with a mechanism involving a critical crack velocity and rates of elastic energy dissipation [16]. For slow crack propagation, the fracture surface of the BMG appears remarkably flat and shining and is featureless when examined with a normal SEM [see Fig. 1(b)] or an optical microscope. As shown in Fig. 1(c), massive shear bands form in the region just near the notch edge (zone II), showing evidence for localized softening and shear instability [17]. Some riverlike shear bands, parallel to the direction of crack propagation, become unstable and bifurcate with increasing distance from the notch site, indicating the branching and healing mechanisms contributing to toughness.

However, when zone I was carefully investigated using HRSEM, damage microvoids at the 100-nm scale are observed as shown in Fig. 2(a). The dimple structure is strikingly similar to that of other BMGs fractured under similar conditions as shown in Fig. 2(b) for Ce-based BMG $\left(K_{C} \sim\right.$ $10 \mathrm{MPa} \sqrt{\mathrm{m}}$ ) and in Fig. 2(c) [10] for tough Ti-based $\left(K_{C} \sim 50 \mathrm{MPa} \sqrt{\mathrm{m}}\right) \mathrm{BMG}$, and the difference actually re- sides in the length scales. The characteristic fracture morphologies in metallic glasses were successfully simulated by Argon and Salama [18] using a grease model. The evidence of microvoids formation and coalescence are also demonstrated by the blunted crack tip in the BMG [Fig. 2(a)]. In this case, the stress concentration is generally not large enough to break bonds at the tip, even though it might be sufficient to generate plasticity in the material surrounding the crack. Such general plasticity is thought to be the cause of the microvoid growth failure. HRSEM

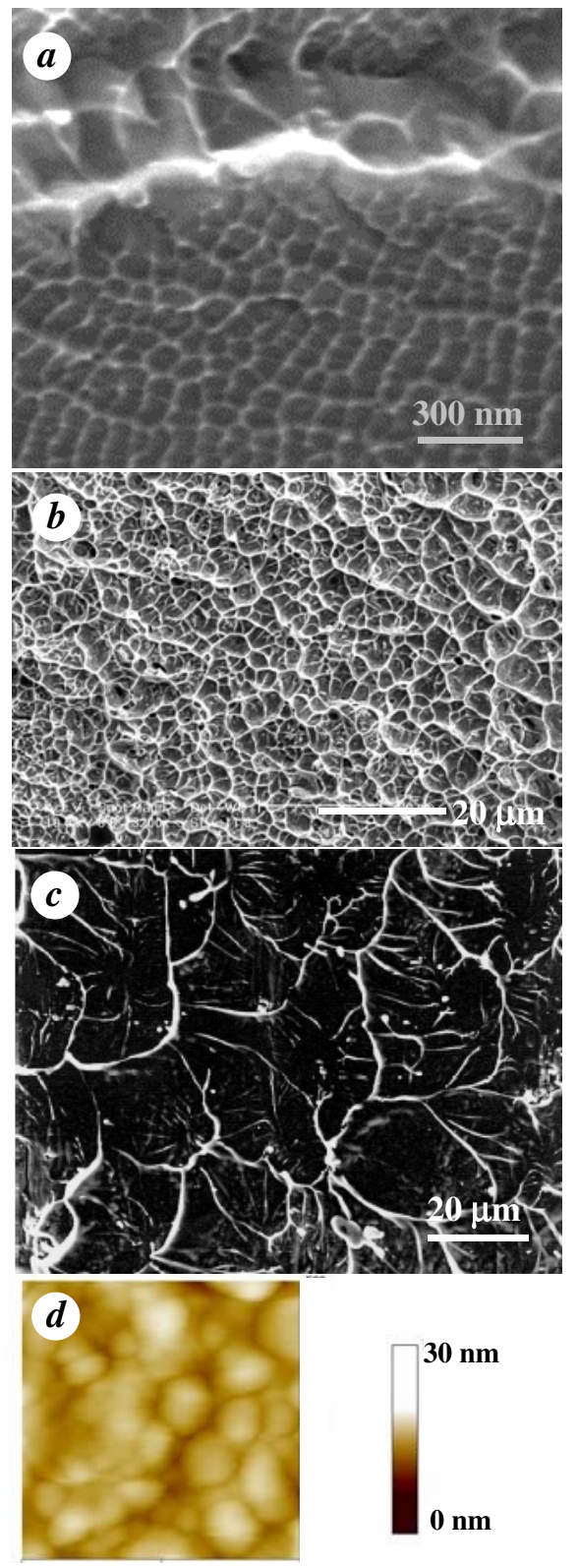

FIG. 2 (color online). High magnification SEM image of the fracture surface; (a) nanometer-scale "dimple" structure of the Mg-based BMG. The upper part shows the structure of river patterned shear bands, demonstrating the coalescence of damage voids, (b) Ce-based BMG, (c) Ti-based BMG, and (d) an AFM image (image size: $500 \mathrm{~nm} \times 500 \mathrm{~nm}$ ) of vein pattern corresponding to (a). 
images of mating fracture surfaces on both sides of the crack further reveal that the veins match in a peak-to-peak fashion. In this type of dynamic crack propagation a large number of voids form rapidly at the crack tip and the material between the voids necks down. The nm-scale dimple structure in the fracture surfaces of the BMG is indicative of the activation of plastic flow processes, possibly as the result of local softening mechanism [18-20]. Figure 2(d) shows an AFM image of the morphology of zone I, which confirms that the size of the cellular structured plastic zone is about $100 \mathrm{~nm}$. The fracture surface roughness $R=h / W$ (where $h$ and $W$ are the depth and the width of the dimple, respectively) [21], which can be used to characterize the ductility of a material, is estimated from the AFM image. For Mg-based BMG, $R$ is about 0.05, while for the Zr-based BMGs, $R>0.5$ [14]. The size of the plastic process zone $w$ can also be estimated by using the Argon-Salama model [18]. For the Mg-based BMG, the $E$, the fracture stress $\sigma_{Y}$, and the surface energy per unit area $\gamma$ are $47.6 \mathrm{GPa}, \sim 700 \mathrm{MPa}$, and $\sim 1.0 \mathrm{~J} / \mathrm{m}^{2}$, respectively. The $w$ of the BMG is estimated to be $\sim 250 \mathrm{~nm}$, which is comparable to the experimental observation. Therefore, both HRSEM and AFM observations show evidence of substantial local necking and plastic deformation at the $100-\mathrm{nm}$ scale. Similar fracture experiments performed in other brittle BMGs such as Fe-based BMGs reveal a similar dimple structure, suggesting that the existence of damage microvoids does not depend on the chemical composition of the studied BMG. The nucleation of microvoids should be related to the glassy structure, which contains an amount of free volumes and inherent atomic density fluctuations at the nm scale [10]. However, it is noted that this shear softening (in marked contrast to the work hardening exhibited by crystalline metals) prevents stable plastic elongation in tension. Thus the BMGs showing plasticity cannot properly be termed ductile, though they are malleable and can be bent plastically [7].

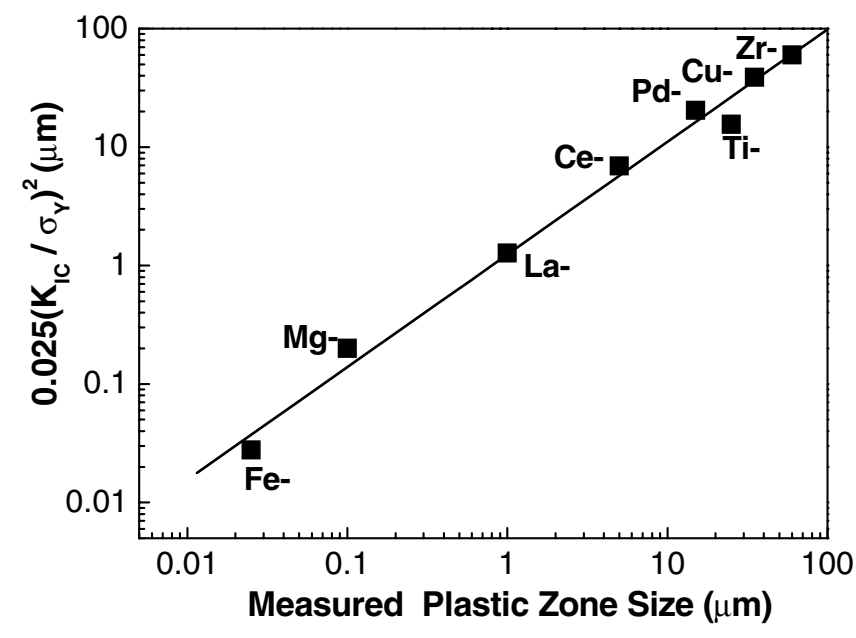

FIG. 3. Fracture toughness or strength response to the plastic process zone size. The details of the glassy materials are listed in Table I.
Previous studies reveal that the local plastic zones in the fracture surface of a variety of BMGs under single-edge notched tension or bending have a strikingly similar shape but at different length scales [7,8,10-14,22,23]. It is interesting to link the average critical plastic zone size $w$ of different glasses exhibiting different degrees of toughness with $K_{C}$. In fact, there is considerable evidence that the $w$ in glassy polymers can be correlated with $\left(K_{C} / \sigma_{Y}\right)^{2}$ as in [15]: $w=\frac{1}{6 \pi}\left(\frac{K_{C}}{\sigma_{Y}}\right)^{2}$. Figure 3 shows the relationship between $\left(K_{C} / \sigma_{Y}\right)^{2}$ and $w$ of BMGs. All the selected materials exhibit the characteristic vein pattern in different length scales in the dominant fracture surface regions. The $w$ measured as the average width of the dimple (or the wavelength of the vein features) of these glasses as single edge notched by tension or bending is estimated directly from the SEM morphology of their fracture surfaces. The $w$ ranges from the nm scale in brittle $\mathrm{MgCuTb} \mathrm{BMG}$ to the $\mu \mathrm{m}$ scale in tough $\mathrm{Zr}$-based BMGs. The values of $K_{C}$ and $\sigma_{Y}$ for the metallic glasses are listed in Table I. Figure 3 shows that $w$ increases linearly with the increase of $\left(K_{C} / \sigma_{Y}\right)^{2}$. Clearly, a correlation exists between $w$ and the toughness of various BMGs. Such a correlation also exists in the same sample measured at different temperatures. In the PdNiP metallic glass, both the $K_{C}$ and the $w$ decrease upon temperature and show a similar correlation [25]. The result indicates that the toughness of BMGs is closely associated with the scale of the $w$ local plastic zone. The correlation permits a direct estimation of the $K_{C}$ based on the yield strength and the $w$ critical plastic zone size of the fracture surface of a BMG.

To understand the correlation, it is important to link the local plastic zone with the crack tip opening displacement (CTOD) and stress intensity. This would also tie together the local behavior with more macroscopic measurements of toughness. The nm-scale dimple structure in the fracture surfaces of the brittle BMG illustrates very local plasticity. The local plasticity contributes very little to the global toughness because it is so localized and the CTOD needed to achieve this plasticity is also very small. This corresponds to the low toughness since toughness scales with CTOD. Tough metallic glasses, on the other hand, exhibit a much larger scale of local plasticity as well as significant crack bifurcation and branching. These lead to a much higher CTOD and hence toughness. The correlation also indicates that, for metallic glasses, the failure at the microscopic level does not depend on the stress and strain at a point but involves a characteristic length related to the microstructure. As long as the cracks are within the process zone or the dimension size of the sample is less than the characteristic length, they do not produce significant additional deformation of the material, and the appearance of the fracture surface formed should show few effects of macroplasticity [26]. The phenomena have been observed in $\mathrm{Zr}$-based metallic glasses where thin plates show ductility in bending while thick plates of the same composition fracture under similar loading [27]. The ribbons 
TABLE I. Data of measured $w, K_{C}$, and $\sigma_{Y}$ of the metallic glasses for Fig. 3. The equation $w=0.025\left(K_{C} / \sigma_{Y}\right)^{2}$ was used to calculate the plastic zone size.

\begin{tabular}{lccccc}
\hline \hline \multicolumn{1}{c}{ Metallic glasses } & $w(\mu \mathrm{m})$ & $K_{C}(\mathrm{MPa} \sqrt{\mathrm{m}}$ & $\sigma_{Y}(\mathrm{MPa})$ & Calculated zone size $(\mu \mathrm{m})$ & References \\
\hline $\mathrm{Zr}_{41} \mathrm{Ti}_{14} \mathrm{Cu}_{12.5} \mathrm{Ni}_{10} \mathrm{Be}_{22.5}$ & 60 & 86 & 1800 & 57.1 & {$[7,14]$} \\
$\mathrm{Cu}_{60} \mathrm{Zr}_{20} \mathrm{Hf}_{20} \mathrm{Ti}_{10}$ & 35 & 67 & 1700 & 38.8 & {$[7,13]$} \\
$\mathrm{Ti}_{50} \mathrm{Ni}_{24} \mathrm{Cu}_{20} \mathrm{~B}_{1} \mathrm{Si}_{2} \mathrm{Sn}_{3}$ & 20 & 50 & 2100 & 14.2 & {$[10]$} \\
$\mathrm{Pd}_{77.5} \mathrm{Si}_{16.5} \mathrm{Cu}_{6}$ & 15 & 45 & 1570 & 20.5 & {$[24]$} \\
$\mathrm{Ce}_{60} \mathrm{Al}_{20} \mathrm{Ni}_{10} \mathrm{Cu}_{10}$ & 5 & 10 & 600 & 6.9 & \\
$\mathrm{La}_{55} \mathrm{Al}_{25} \mathrm{Ni}_{5} \mathrm{Cu}_{10} \mathrm{Co}_{5}$ & 1 & 5 & 700 & 1.3 & {$[12]$} \\
$\mathrm{Mg}_{65} \mathrm{Cu}_{25} \mathrm{~Tb}_{10}$ & 0.1 & 2 & 660 & 0.2 & {$[22]$} \\
$\mathrm{Fe}_{46} \mathrm{Ni}_{32} \mathrm{~V}_{2} \mathrm{Si}_{14} \mathrm{~B}_{6}$ & 0.02 & 4 & 3800 & 0.028 & \\
\hline \hline
\end{tabular}

$(\sim 100 \mu \mathrm{m})$ even can be bent through $180^{\circ}$. Thus, it is reasonable to believe that thin films of $\mathrm{MgCuTb}$ glass would be much tougher when it is less than $1 \mu \mathrm{m}$ thick.

An issue of current interest is the possibility of plastic deformation at crack tips in silicate glasses at room temperature. A recent AFM study reported the formation of plastic zones of $\mathrm{nm}$ scales in silicate glass undergoing fracture [28]. However, a subsequent study of silicate glasses under a similar situation did not observe such cavities [5]. Although the brittleness of the studied Mgbased BMG approaches that of silicate glasses, the observed fracture softening mechanism of BMGs should not be generalized to other types of brittle glasses without further investigations. For instance, the silicate glasses do not show vein patterns on the fracture surfaces, and the peaks on one side of the fracture surface fit the valleys on the mating fracture surface consistent with the idea of completely brittle rupture [5]. This is very different from the observed features of the brittle BMG.

In summary, we have observed experimentally the formation of nm-scale damage microvoids in the fracture surface of the brittle Mg-based BMG. This suggests that plastic deformation occurs during fracture far below the glass transition temperature. A clear correlation is established between the fracture toughness and the length scale of the plastic process zone for various brittle and tough BMGs. This indicates that the fracture in brittle BMGs might proceed through fracture softening mechanism, and the fracture can be regarded as a flow process but at different length scales. For applications, such an understanding could guide the design of metallic glasses with enhanced plasticity. The observed effect of the length scale could be especially important for $\mathrm{mm}$ - and $\mathrm{nm}$-scale fabrication of metallic glass structures such as manufacturing metallic glass foams by modulating wall thickness [29].

We thank A. L. Greer for a critical reading of the manuscript and the support of the NSF, China (Grants No. 10328408 and No. 50321101) and Reference Metals (for J. J. L.).
*Corresponding author.

Electronic address: whw@aphy.iphy.ac.cn

[1] J. Fineberg and M. Marde, Phys. Rep. 313, 1 (1999).

[2] Z. F. Zhang, J. Eckert, and L. Schultz, Phys. Rev. Lett. 91, 045505 (2003).

[3] A. Kelly, W. R. Tyson, and A. H. Cottrell, Philos. Mag. 15, 576 (1967).

[4] J. R. Rice and R. Thomson, Philos. Mag. 29, 73 (1974).

[5] J-P. Guin and S. M. Wiederhorn, Phys. Rev. Lett. 92, 215502 (2004).

[6] X. K. Xi and W. H. Wang, J. Non-Cryst. Solids 344, 105 (2004).

[7] J. J. Lewandowski, W. H. Wang, and A. L. Greer, Philos. Mag. Lett. (to be published).

[8] P. Lowhaphandu and J. J. Lewandowski, Scr. Mater. 38, 1811 (1998).

[9] S. M. Wiederhorn, J. Am. Ceram. Soc. 52, 99 (1969).

[10] A. Inoue, Acta Mater. 48, 279 (2000); Mater. Sci. Eng. A 304, 1 (2001).

[11] W. H. Wang et al., Mater. Sci. Eng., R 44, 45 (2004).

[12] N. Nagendra et al., Acta Mater. 48, 2603 (2000).

[13] P. Wesseling et al., Scr. Mater. 51, 151 (2004).

[14] R. D. Conner et al., Scr. Mater. 37, 1373 (1997).

[15] A. Christiansen and J. B. Shortall, J. Mater. Sci. 11, 1113 (1976).

[16] E. Sharon et al., Phys. Rev. B 54, 7128 (1996).

[17] F. A. Spaepen, Acta Metall. 25, 407 (1977).

[18] A. S. Argon and M. Salama, Mater. Sci. Eng. A 23, 219 (1976).

[19] K. M. Flores and R. H. Dauskardt, J. Mater. Res. 11, 638 (1999).

[20] P. G. Fox and K. N. G. Fuller, Nature (London), 233, 13 (1971).

[21] A. W. Thompson and M.F. Ashby, Scr. Metall. 18, 127 (1984).

[22] C. H. Shek et al., J. Non-Cryst. Solids 224, 244 (1998).

[23] D. M. Kulawansa et al., J. Mater. Res. 8, 2543 (1993).

[24] H. Kimura and T. Masumoto, Acta Metall. 28, 1677 (1980).

[25] S. Takayama and R. Maddin, Philos. Mag. 32, 457 (1975).

[26] A. Tatschl et al., J. Mater. Res. 15, 898 (2000).

[27] R. D. Conner et al., Acta Mater. 52, 2429 (2004).

[28] F. Celarie et al., Phys. Rev. Lett. 90, 075504 (2003).

[29] R. D. Conner et al., J. Appl. Phys. 94, 904 (2003). 